\title{
Spectral density of dense random networks and the breakdown of the Wigner semicircle law
}

\author{
Fernando L. Metz $\odot^{*}$ \\ Physics Institute, Federal University of Rio Grande do Sul, 91501-970 Porto Alegre, Brazil \\ and London Mathematical Laboratory, 18 Margravine Gardens, London W6 8RH, United Kingdom \\ Jeferson D. Silva (1) \\ Physics Institute, Federal University of Rio Grande do Sul, 91501-970 Porto Alegre, Brazil
}

(Received 30 June 2020; accepted 6 October 2020; published 22 October 2020)

\begin{abstract}
Although the spectra of random networks have been studied for a long time, the influence of network topology on the dense limit of network spectra remains poorly understood. By considering the configuration model of networks with four distinct degree distributions, we show that the spectral density of the adjacency matrices of dense random networks is determined by the strength of the degree fluctuations. In particular, the eigenvalue distribution of dense networks with an exponential degree distribution is governed by a simple equation, from which we uncover a logarithmic singularity in the spectral density. We also derive a relation between the fourth moment of the eigenvalue distribution and the variance of the degree distribution, which leads to a sufficient condition for the breakdown of the Wigner semicircle law for dense random networks. Based on the same relation, we propose a classification scheme of the distinct universal behaviors of the spectral density in the dense limit. Our theoretical findings should lead to important insights on the mean-field behavior of models defined on graphs.
\end{abstract}

DOI: 10.1103/PhysRevResearch.2.043116

\section{INTRODUCTION}

A random network or graph is a collection of nodes joined by edges following a probabilistic rule. Random networks are formidable tools to model large assemblies of interacting units, like neurons in the brain, computers and routers on the internet, or persons forming a friendship network [1]. Motivated by our increasing ability to collect and process vast amounts of empirical data, the theory of random networks has experienced enormous progress, leading to important insights in physics, biology, and sociology [2]. The implications of the structure of networks to the dynamical processes occurring on them remains a fundamental topic in network theory [3].

Dynamical processes on a random network are to a large extent governed by the spectrum of the corresponding adjacency random matrix. This is a random matrix where each entry equals the strength of the interaction between a pair of nodes. The study of a broad range of problems amounts to linearizing a large set of differential equations [4-6], coupled through a random network, around a stationary state, whose stability and transient dynamics are ultimately determined by the eigenvalue distribution of the adjacency matrix. Examples in this context are the study of the epidemic threshold for

\footnotetext{
*fmetzfmetz@gmail.com

Published by the American Physical Society under the terms of the Creative Commons Attribution 4.0 International license. Further distribution of this work must maintain attribution to the author(s) and the published article's title, journal citation, and DOI.
}

the spreading of diseases [7], the synchronization transition in networks of coupled oscillators [8,9], and the functional stability of large biological systems, such as gene regulatory networks [10,11], ecosystems [12-14], and neural networks [15-17]. The spectrum of the adjacency matrix also contains information about the network structure, since the trace of the adjacency matrix raised to a certain power yields the number of network loops of a given length [18]. Therefore, the problem of how the network architecture influences the spectrum of the adjacency matrix has attracted considerable research effort [4,19-25].

Synthetic models of random networks provide a controlled way to study the role of the network structure on the spectrum of the adjacency matrix. The degree sequence is the most basic tool to characterize the graph structure [1]. The degree of a node counts the number of edges attached to the node, while the degree sequence specifies all network degrees. When a network has an infinitely large number of constituents, it is natural to consider the degree distribution, i.e., the fraction of nodes with a certain degree, instead of dealing with the degree sequence. The configuration model stands out as one of the most fundamental and versatile models of random graphs [1,26-28], since it enables the degree distribution to be freely specified while keeping the pattern of interconnections entirely random. From a practical viewpoint, the configuration model resolves one of the main shortcomings of ErdösRényi random graphs [29-31], namely, its Poisson degree distribution, which has little resemblance to the long-tailed degree distributions found in empirical networks $[32,33]$. The priceless possibility to fix the degree distribution of a random graph is not only useful to model the structure of empirical 
networks but offers the ideal setting to examine the impact of degree fluctuations on the spectrum of the adjacency matrix.

There has been a significant amount of numeric and analytic work on the spectral properties of adjacency random matrices [4,19-25,34-41]. A remarkable analytic result is the set of exact and mathematically rigorous equations determining the eigenvalue distribution of the configuration model [36-38,42]. These equations form a natural starting point to study the impact of degree fluctuations on network spectra. Unfortunately, aside from a few particular cases [24,39,42], these equations can be analytically solved only in the dense limit [36,37], when the mean degree becomes infinitely large and random networks approach a fully connected structure.

The analytic solution for the dense limit of the aforementioned equations is at the root of perturbative and nonperturbative approximations for the eigenvalue distribution of large-degree random networks [20,34,43]. With the exception of graphs with a power-law degree distribution $[19,21,35]$ whose moments are divergent, one expects that the eigenvalue distribution of undirected random networks converges, in the dense limit, to the Wigner semicircle distribution of random matrix theory, reflecting a high level of universality. This expectation has been confirmed numerically [19] and analytically [36,37] for Erdös-Rényi and regular random graphs. However, recent work [44] has rigorously shown that the spectrum of the configuration model does depend on the degree distribution in the dense limit. The main theorem in Ref. [44] also provides an approach to compute the spectral density for specific degree distributions. In spite of these rigorous results, a more detailed understanding of how the structure of a dense random network influences its spectrum, and the universal status of the Wigner semicircle law, is still lacking.

In this paper, we study the dense limit of the eigenvalue distribution of undirected networks drawn from the configuration model. We analyze four examples of degree distributions in which all moments are finite and the variances scale differently with the average degree, and we show that the dense limit of the eigenvalue distribution is determined by the degree fluctuations. In particular, we derive an equation yielding the eigenvalue distribution of dense random graphs with an exponential degree distribution [28], from which we unveil the existence of a logarithmic divergence in the spectral density and the absence of sharp spectral edges, i.e., the eigenvalue distribution of exponential random graphs is supported on the entire real line. These are remarkable differences with respect to the Wigner semicircle distribution of random matrix theory [45]. We also discuss how the analytic equations for the spectral density can be derived in two different ways: from the dense limit of the exact resolvent equations in Refs. [36,37,42] and from the main theorem proved in Ref. [44]. Based on an exact calculation of the fourth moment of the eigenvalue distribution, we obtain a sufficient condition for the breakdown of the Wigner semicircle law. This condition is given only in terms of the variance of the degree distribution. We finish the paper by proposing a classification of the different universal behaviors of the eigenvalue distribution of the configuration model in the dense limit, based on an exponent characterizing how the variance of the degree distribution diverges for increasing mean degrees.
Our paper is organized as follows. In the next section, we define the adjacency matrix of random networks and the spectral density. Section III introduces the configuration model and defines the degree distributions studied in this paper. In Sec. IV, we present numeric and analytic results for the dense limit of the spectral density for each example of degree distribution. Section IV explains how the analytic equations for the spectral density are obtained from the dense limit of the resolvent equations, while in Sec. V we derive these equations from the main theorem in Ref. [44]. The condition for the breakdown of the Wigner semicircle law and the classification of the different universal behaviors are discussed in Sec. VI. We summarize our results and conclude in Sec. VII.

\section{THE ADJACENCY MATRIX OF RANDOM NETWORKS}

Let $N$ be the total number of network nodes. The set of binary random variables $\left\{C_{i j}\right\}_{i, j=1, \ldots, N}$ specifies the network structure: If $C_{i j}=1$, there is an undirected link $i \leftrightarrow j$ between nodes $i$ and $j$, while $C_{i j}=0$ means this link is absent. We also associate a random weight $J_{i j} \in \mathbb{R}$ to each edge $i \leftrightarrow j$, which accounts for the interaction strength between nodes $i$ and $j$. We consider undirected simple random networks that have no self-edges nor multiedges, in which $C_{i j}=C_{j i}, J_{i j}=J_{j i}$, and $C_{i i}=0$.

The degree $K_{i}=\sum_{j=1}^{N} C_{i j}$ of node $i$ is a random variable that counts the number of nodes attached to $i$, while the degree sequence $K_{1}, \ldots, K_{N}$ provides global information on the fluctuations of the network connectivity. In the limit $N \rightarrow \infty$, it is more convenient to work with the degree distribution

$$
p_{k}=\lim _{N \rightarrow \infty} \frac{1}{N} \sum_{i=1}^{N} \delta_{k, K_{i}},
$$

where $\delta$ is the Kronecker symbol. The average degree $c$ and the variance $\sigma^{2}$ of $p_{k}$ read

$$
c=\sum_{k=0}^{\infty} k p_{k}, \quad \sigma^{2}=\sum_{k=0}^{\infty} p_{k}(k-c)^{2} .
$$

The degree distribution $p_{k}$ is one of the primary quantities to characterize the structure of random networks in the limit $N \rightarrow \infty$.

We will study the eigenvalue distribution of the $N \times N$ symmetric adjacency matrix $\boldsymbol{A}$, with elements defined as

$$
A_{i j}=\frac{C_{i j} J_{i j}}{\sqrt{c}} .
$$

The adjacency matrix fully encodes the network structure, along with the coupling strengths between adjacent nodes. The empirical spectral measure of $\boldsymbol{A}$ is given by

$$
\rho_{N}(\lambda)=\frac{1}{N} \sum_{\alpha=1}^{N} \delta\left(\lambda-\lambda_{\alpha}\right),
$$

where $\lambda_{1}, \ldots, \lambda_{N}$ are the (real) eigenvalues of $\boldsymbol{A}$. By introducing the $N \times N$ resolvent matrix

$$
\boldsymbol{G}(z)=(z-\boldsymbol{A})^{-1},
$$

with $z=\lambda-i \eta$ on the lower half complex plane, the empirical spectral measure follows from the diagonal elements 
TABLE I. The analytic expression for the degree distribution $p_{k}$ and the corresponding variance $\sigma^{2}$ in the case of regular, Poisson, exponential, and Borel random networks. The Borel degree distribution is defined for $c>1$, while the other three distributions are defined for $c>0$. The Borel degree distribution is supported in $k \geqslant 1$, the degree distribution of regular graphs is supported at $k=c$, and both Poisson and exponential degree distributions are supported in $k \geqslant 0$.

\begin{tabular}{ccccc}
\hline \hline & Regular & Poisson & Exponential & Borel \\
\hline$p_{k}$ & $\delta_{k, c}$ & $\frac{e^{-c} c^{k}}{k !}$ & $\frac{1}{c+1}\left(\frac{c}{c+1}\right)^{k}$ & $\frac{e^{-\frac{(c-1)}{c} k}}{k !}\left[\frac{k(c-1)}{c}\right]^{k-1}$ \\
$\sigma^{2}$ & 0 & $c$ & $c^{2}+c$ & $c^{3}-c^{2}$ \\
\hline \hline
\end{tabular}

of $\boldsymbol{G}(z)$ :

$$
\rho_{N}(\lambda)=\frac{1}{\pi N} \lim _{\eta \rightarrow 0^{+}} \sum_{i=1}^{N} \operatorname{Im} G_{i i}(z) .
$$

In the limit $N \rightarrow \infty$, the empirical mean of $\operatorname{Im} G_{i i}(z)$ normally converges to its ensemble-averaged value, obtained from the distribution of $\boldsymbol{A}$, which implies that $\rho(\lambda)=\lim _{N \rightarrow \infty} \rho_{N}(\lambda)$ is well-defined. Here we will study the spectral density $\rho(\lambda)$ when the average degree grows to infinity, hence the limit $c \rightarrow$ $\infty$ is performed after $N \rightarrow \infty$. The scaling of the elements $A_{i j}$ with the average degree $c$ in Eq. (2) ensures the spectral density has a finite variance when $c \rightarrow \infty$.

\section{THE CONFIGURATION MODEL OF NETWORKS}

We study random networks with arbitrary degree distributions, known as the configuration model of networks [1,26-28], where $p_{k}$ is specified at the outset. A single instance of the adjacency matrix of the configuration model is created as follows. First, the degrees $K_{1}, \ldots, K_{N}$ are drawn independently from a common distribution $p_{k}$. After assigning $K_{i}$ stubs of edges to each node $i(i=1, \ldots, N)$, a pair of stubs is uniformly chosen at random and then connected to form an edge. This last step is repeated on the remainder stubs until there are no stubs left, and the outcome is a particular matching of the stubs with the prescribed random degrees $K_{1}, \ldots, K_{N}$. We do not allow for the existence of self-edges and multiedges in the random network. We set $A_{i j}=J_{i j} / \sqrt{c}$ if there is an edge connecting nodes $i$ and $j$, and $A_{i j}=0$ otherwise. The coupling strengths $\left\{J_{i j}\right\}_{i, j=1, \ldots, N}$ are independently and identically distributed random variables drawn from a common distribution $P_{J}$. We refer to Ref. [1] for other properties and subtleties of the configuration model. In the limit $N \rightarrow \infty$, the ensemble of adjacency random matrices $\boldsymbol{A}$ constructed from this procedure is specified by the degree distribution $p_{k}$ and the distribution of weights $P_{J}$. This is probably the simplest network model that allows us to clearly exploit the influence of degree fluctuations on the spectral properties of $\boldsymbol{A}$.

We will present results for regular, Poisson, exponential, and Borel degree distributions. The analytic expression for $p_{k}$ in each case, together with the variance $\sigma^{2}$, are displayed in Table I. The properties of the configuration model with Poisson and exponential degree distributions have been extensively discussed in Refs. [1,28]. The degree distributions

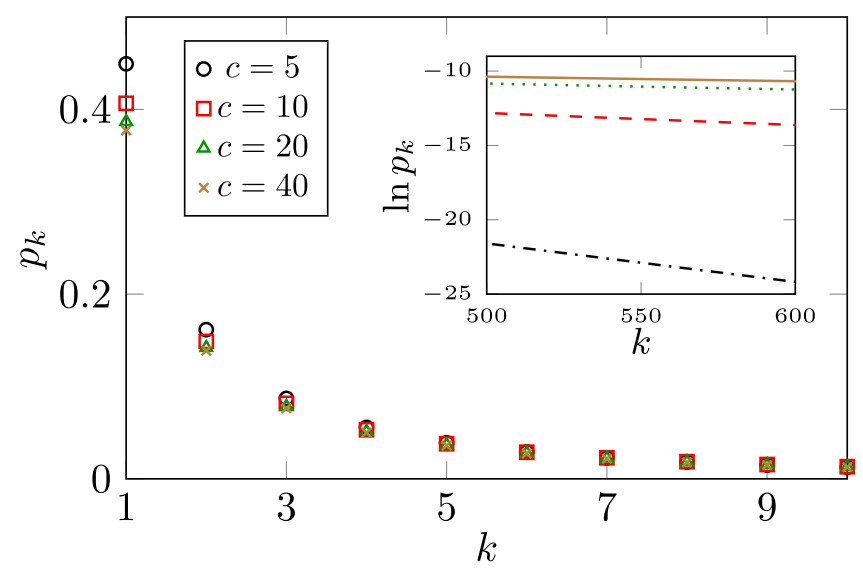

FIG. 1. The Borel degree distribution $p_{k}$ (see Table I) for different values of the average degree $c$. The inset depicts the exponential tail of $p_{k}$ for large $k$. The values of $c$ in the inset are $c=40$ (solid line), $c=20$ (dotted line), $c=10$ (dashed line), and $c=5$ (dotdashed line).

in Table I obey the following properties: (i) $p_{k}$ is parametrized solely in terms of the mean degree $c$, (ii) all moments of $p_{k}$ are finite for $c<\infty$, and (iii) for sufficiently large $c$, the variances obey $\sigma_{\text {reg }}^{2}<\sigma_{\text {pois }}^{2}<\sigma_{\text {exp }}^{2}<\sigma_{\text {bor }}^{2}$. These four examples of degree distributions are very convenient to study, in a controllable way, the effect of degree fluctuations on random networks as we increase $c$. Indeed, we can compare for $c \gg 1$ the spectral density of networks with the same average degree, but with increasing variances $\sigma^{2}$. We do not consider here random networks with power-law degree distributions $[32,33]$, since in this case higher-order moments of $p_{k}$ already diverge for finite $c$.

Although the Borel degree distribution is not commonly employed in the study of random networks, the rate of its exponential decay is smaller than the one of the exponential degree distribution with the same $c$, which makes the Borel distribution well-suited to our analysis. The Borel distribution, introduced in the context of queuing theory [46,47], also appears as the distribution of the total progeny in a GaltonWatson branching process with Poisson distributed degrees [48]. Figure 1 illustrates the Borel degree distribution for different average degrees. For fixed $k \ll c$, the Borel distribution $p_{k}$ attains a finite limit as $c \rightarrow \infty$, in contrast to the Poisson and the exponential degree distribution. For $k=O(c)$, the Borel distribution $p_{k}$ is proportional to $1 / \sqrt{c}$ for $c \gg 1$. Therefore, Borel networks with large $c$ contain a finite fraction of nodes with $c$-independent degrees and a smaller fraction of nodes with degrees proportional to $c$. Note also that $p_{k}$ in Fig. 1 is highly skewed, its mode is located at $k=1$, and the tail of $p_{k}$ decays as $\ln p_{k} \sim\left(\frac{1}{c}+\ln \left(\frac{c-1}{c}\right)\right) k$ for $k \gg 1$. All network models considered here have exponentially decaying degree distributions.

\section{THE DENSE LIMIT OF THE SPECTRAL DENSITY}

The configuration model of networks has a key property: In the limit $N \rightarrow \infty$, the set of nodes in the neighborhood of a given node, drawn uniformly from the network, is arranged in a treelike structure [42]. Nevertheless, the topology 
of random networks is fundamentally different from a Cayley tree [49] in the sense that boundary nodes are absent from the configuration model and cycles do survive in the limit $N \rightarrow$ $\infty$, but their average length scales as $\ln N$ for $N \gg 1$. Roughly speaking, a random network can be seen as a Cayley tree with fluctuating degrees, wrapped onto itself.

In the limit $N \rightarrow \infty$, the spectral density is obtained from

$$
\rho(\lambda)=\frac{1}{\pi} \lim _{\eta \rightarrow 0^{+}} \int_{\operatorname{Im} g \geqslant 0} d^{2} g \mathcal{P}(g) \operatorname{Im} g,
$$

where $d^{2} g \equiv d \operatorname{Re} g d \operatorname{Im} g$, and $\mathcal{P}(g)$ is the joint distribution of the real and imaginary parts of $G_{i i}(z)$. The symbol $\int_{\operatorname{Im} g \geqslant 0}$ represents an integral over $g \in \mathbb{C}$ restricted to the upper-half complex plane. The distribution of the resolvent follows from the solution of the coupled equations $[36,37]$

$$
\begin{aligned}
\mathcal{P}(g)= & \sum_{k=0}^{\infty} p_{k} \int_{\operatorname{Im} g \geqslant 0}\left[\prod_{\ell=1}^{k} d^{2} g_{\ell} \mathcal{P}_{\text {cav }}\left(g_{\ell}\right)\right] \\
& \times\left\langle\delta\left[g-\left(\frac{1}{z-\frac{1}{c} \sum_{\ell=1}^{k} J_{\ell}^{2} g_{\ell}}\right)\right]\right\rangle_{J_{1}, \ldots, J_{k}}, \\
\mathcal{P}_{\text {cav }}(g)= & \sum_{k=0}^{\infty} \frac{k p_{k}}{c} \int_{\operatorname{Im} g \geqslant 0}\left[\prod_{\ell=1}^{k-1} d^{2} g_{\ell} \mathcal{P}_{\text {cav }}\left(g_{\ell}\right)\right] \\
& \times\left\langle\delta\left[g-\left(\frac{1}{z-\frac{1}{c} \sum_{\ell=1}^{k-1} J_{\ell}^{2} g_{\ell}}\right)\right]\right\rangle_{J_{1}, \ldots, J_{k-1}},
\end{aligned}
$$

where $\langle\ldots\rangle_{J_{1}, \ldots, J_{L}}$ denotes the average over the independent interaction strengths $J_{1}, \ldots, J_{L}$, distributed according to $P_{J}$. The quantity $\mathcal{P}_{\text {cav }}(g)$ is the joint distribution of the real and imaginary parts of the resolvent elements $G_{i i}^{(j)}(z)$ on the cavity graph [36,50], defined as the graph where an arbitrary node $j$ and all its edges have been removed. Equations (7) and (8) have been derived using both the cavity $[36,50]$ and the replica method [37] of disordered systems, based on the local treelike structure of the configuration model. Equations (6)-(8) have been rigorously proven in Ref. [42] and they are exact in the limit $N \rightarrow \infty$, constituting an interesting point of departure to study the dense limit $c \rightarrow \infty$ of the spectral density $\rho(\lambda)$.

Let $F(G)$ be an arbitrary function of the complex random variable $G$ distributed as $\mathcal{P}_{\text {cav }}(g)$. By defining the average of $F(G)$,

$$
\langle F(G)\rangle=\int_{\operatorname{Im} g \geqslant 0} d^{2} g \mathcal{P}_{\text {cav }}(g) F(g),
$$

we can use Eq. (8) and rewrite the above expression as

$$
\langle F(G)\rangle=\int_{\operatorname{Im} q \geqslant 0} d^{2} q \mathcal{W}_{\text {cav }}(q) F\left(\frac{1}{z-q}\right),
$$

where we introduced the distribution of $Q^{\prime} \equiv \frac{1}{c} \sum_{\ell=1}^{K-1} J_{\ell}^{2} G_{\ell}$

$$
\begin{aligned}
\mathcal{W}_{\text {cav }}(q)= & \sum_{k=0}^{\infty} \frac{k p_{k}}{c} \int_{\operatorname{Im} g \geqslant 0}\left[\prod_{\ell=1}^{k-1} d^{2} g_{\ell} \mathcal{P}_{\text {cav }}\left(g_{\ell}\right)\right] \\
& \times\left\langle\delta\left(q-\frac{1}{c} \sum_{\ell=1}^{k-1} J_{\ell}^{2} g_{\ell}\right)\right\rangle_{J_{1}, \ldots, J_{k-1}},
\end{aligned}
$$

with the random variable $K$ denoting the degree of an uniformly chosen node. Following an analogous procedure, we can also rewrite the spectral density in terms of the distribution $\mathcal{W}(q)$ of the random variable $Q \equiv \frac{1}{c} \sum_{\ell=1}^{K} J_{\ell}^{2} G_{\ell}$,

$$
\rho(\lambda)=\frac{1}{\pi} \lim _{\eta \rightarrow 0^{+}} \int_{\operatorname{Im} q \geqslant 0} d^{2} q \mathcal{W}(q) \operatorname{Im}\left(\frac{1}{z-q}\right),
$$

defined as

$$
\begin{aligned}
\mathcal{W}(q)= & \sum_{k=0}^{\infty} p_{k} \int_{\operatorname{Im} g \geqslant 0}\left[\prod_{\ell=1}^{k} d^{2} g_{\ell} \mathcal{P}_{\text {cav }}\left(g_{\ell}\right)\right] \\
& \times\left\langle\delta\left(q-\frac{1}{c} \sum_{\ell=1}^{k} J_{\ell}^{2} g_{\ell}\right)\right\rangle_{J_{1}, \ldots, J_{k}} .
\end{aligned}
$$

We note that $\mathcal{W}(q)$ and $\mathcal{W}_{\text {cav }}(q)$ are distributions of sums of independent complex random variables containing a random and large number of terms. For the examples of $p_{k}$ inTable I, one can show that, in the limit $c \rightarrow \infty$, the number of summands in $Q$ and $Q^{\prime}$ diverges. Thus, instead of working with the distributions of the resolvent, it is more convenient to extract the $c \rightarrow \infty$ limit of $\mathcal{W}(q)$ and $\mathcal{W}_{\text {cav }}(q)$. Let us introduce the characteristic functions $\varphi(p, t)$ and $\varphi_{\text {cav }}(p, t)$ of, respectively, $\mathcal{W}(q)$ and $\mathcal{W}_{\text {cav }}(q)$ :

$$
\begin{gathered}
\varphi(p, t)=\sum_{k=0}^{\infty} p_{k} \exp \left[k S_{c}(p, t)\right], \\
\varphi_{\text {cav }}(p, t)=\sum_{k=0}^{\infty} \frac{k p_{k}}{c} \exp \left[(k-1) S_{c}(p, t)\right],
\end{gathered}
$$

with

$$
\begin{aligned}
S_{c}(p, t)= & \ln \left[\int_{\operatorname{Im} g \geqslant 0} d^{2} g \mathcal{P}_{\text {cav }}(g) \int_{-\infty}^{\infty} d x P_{J}(x)\right. \\
& \left.\times \exp \left(-\frac{i p x^{2} \operatorname{Re} g}{c}-\frac{i t x^{2} \operatorname{Im} g}{c}\right)\right] .
\end{aligned}
$$

To study the dense limit $c \rightarrow \infty$, we expand $S_{c}(p, t)$ in powers of $1 / c$, keeping in mind that the moments of $\mathcal{P}_{\text {cav }}(g)$ depend on $c$. The leading term

$$
S_{\infty}(p, t)=-\frac{i\left\langle J^{2}\right\rangle_{J}}{c}\left(p \operatorname{Re}\langle G\rangle_{\infty}+t \operatorname{Im}\langle G\rangle_{\infty}\right)
$$

should yield the dense limit $c \rightarrow \infty$ of the spectral density. Note that we assumed $\langle G\rangle$ converges to a well-defined limit $\langle G\rangle_{\infty}$ when $c \rightarrow \infty$. To proceed further, we specify the degree distribution $p_{k}$ in Eqs. (13) and (14).

\section{A. Regular and Poisson random graphs}

Although it is well known that the dense limit of $\rho(\lambda)$ converges to the semicircle distribution for regular and Poisson random graphs, it is instructive to illustrate our approach for these simple models, characterized by highly peaked degree distributions around the mean value $c$. Substituting the explicit forms of $p_{k}$ (see Table I) in Eqs. (13) and (14), and using the asymptotic behavior of Eq. (16), we obtain

$$
\varphi(p, t)=\varphi_{\text {cav }}(p, t)=e^{-i\left\langle J^{2}\right\rangle_{J}\left(p \operatorname{Re}\langle G\rangle_{\infty}+t \operatorname{Im}\langle G\rangle_{\infty}\right)},
$$




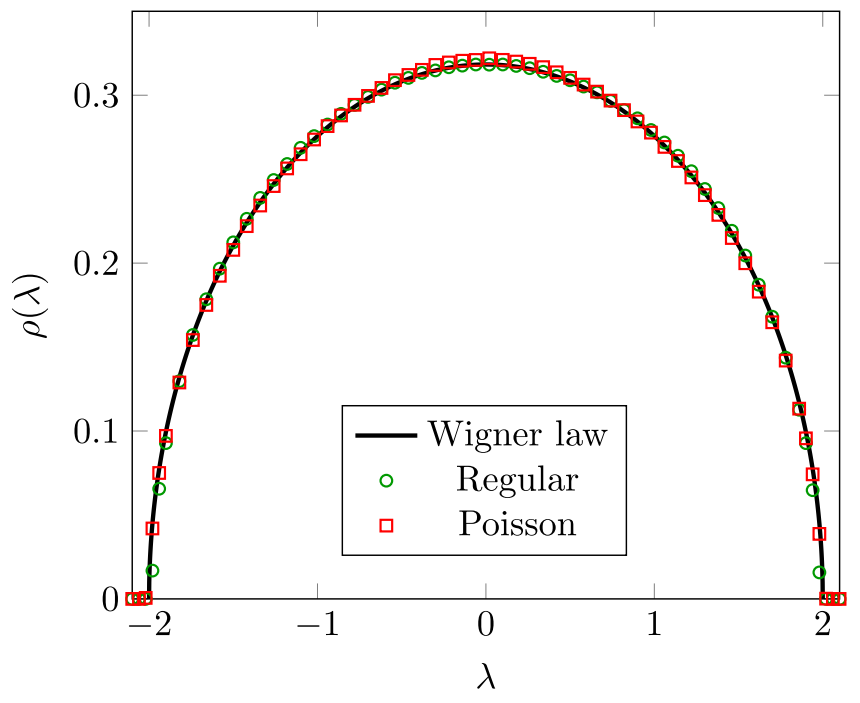

FIG. 2. The dense limit $c \rightarrow \infty$ of the spectral density of regular and Poisson random networks with coupling strengths drawn from $P_{J}(x)=\delta(x-1)$. The solid line represents the Wigner semicircle distribution, Eq. (20), and the symbols are numerical diagonalization results obtained from 100 samples of $10^{4} \times 10^{4}$ adjacency random matrices with $c=100$.

which promptly leads to the Dirac delta distribution in the complex plane:

$$
\mathcal{W}(q)=\mathcal{W}_{\text {cav }}(q)=\delta\left(q-\left\langle J^{2}\right\rangle_{J}\langle G\rangle_{\infty}\right) .
$$

The fact that the resolvent statistics of regular and Poisson random graphs are both described by the same characteristic function, Eq. (17), already demonstrates that these models exhibit the same universal behavior for $c \rightarrow \infty$. The analytic expression for $\mathcal{W}_{\text {cav }}(q)$ allows us to determine $\langle G\rangle_{\infty}$ through Eq. (9), which fulfills

$$
\langle G\rangle_{\infty}=\frac{1}{z-\left\langle J^{2}\right\rangle_{J}\langle G\rangle_{\infty}},
$$

while the spectral density simply follows from Eq. (11):

$$
\rho(\lambda)=\frac{1}{\pi} \lim _{\eta \rightarrow 0^{+}} \operatorname{Im}\left(\frac{1}{z-\left\langle J^{2}\right\rangle_{J}\langle G\rangle_{\infty}}\right) .
$$

By solving the quadratic Eq. (19), we recover the Wigner semicircle law for the Gaussian ensembles of random matrix theory [45]:

$$
\rho_{\mathrm{W}}(\lambda)= \begin{cases}\frac{1}{2 \pi\left\langle J^{2}\right\rangle_{J}} \sqrt{4\left\langle J^{2}\right\rangle_{J}-\lambda^{2}}, & \text { if }|\lambda|<2 \sqrt{\left\langle J^{2}\right\rangle_{J}} \\ 0, & \text { if }|\lambda| \geqslant 2 \sqrt{\left\langle J^{2}\right\rangle_{J}}\end{cases}
$$

Figure 2 compares Eq. (20) with numerical results obtained from diagonalizing large adjacency matrices $\boldsymbol{A}$ with average degree $c=100$. The correspondence between the numerical data and the theoretical expression is excellent for both regular and Poisson random graphs.

\section{B. Exponential random graphs}

In this subsection, we consider the dense limit of exponential random graphs, for which $p_{k}$ decays slower than Poisson graphs for $k \gg 1$ (see Table I). Exponential degree distributions have been observed in some examples of empirical networks, such as the North American power grid [51] and the neural network of the worm C. Elegans [52].

Inserting the expressions for $S_{\infty}(p, t)$ and $p_{k}$ in Eqs. (13) and (14) and taking the limit $c \rightarrow \infty$, we obtain the asymptotic forms

$$
\begin{aligned}
\varphi(p, t) & =\frac{1}{1+i p\left\langle J^{2}\right\rangle_{J} \operatorname{Re}\langle G\rangle_{\infty}+i t\left\langle J^{2}\right\rangle_{J} \operatorname{Im}\langle G\rangle_{\infty}}, \\
\varphi_{\mathrm{cav}}(p, t) & =\frac{1}{\left[1+i p\left\langle J^{2}\right\rangle_{J} \operatorname{Re}\langle G\rangle_{\infty}+i t\left\langle J^{2}\right\rangle_{J} \operatorname{Im}\langle G\rangle_{\infty}\right]^{2}},
\end{aligned}
$$

which already show that the dense limit of $\rho(\lambda)$ is not described by the Wigner semicircle law in this case. The distributions $\mathcal{W}(q)$ and $\mathcal{W}_{\text {cav }}(q)$ are the Fourier transforms of their characteristic functions. Defining

$$
\begin{aligned}
\mathcal{K}(q, \epsilon)= & \int_{-\infty}^{\infty} \frac{d p d t}{4 \pi^{2}} \exp (i p \operatorname{Re} q+i t \operatorname{Im} q) \\
& \times\left[\epsilon+i p\left\langle J^{2}\right\rangle_{J} \operatorname{Re}\langle G\rangle_{\infty}+i t\left\langle J^{2}\right\rangle_{J} \operatorname{Im}\langle G\rangle_{\infty}\right]^{-1}
\end{aligned}
$$

with $\epsilon \geqslant 1$ an auxiliary parameter, $\mathcal{W}(q)$ and $\mathcal{W}_{\text {cav }}(q)$ are obtained from

$$
\mathcal{W}(q)=\mathcal{K}(q, \epsilon=1), \quad \mathcal{W}_{\text {cav }}(q)=-\left.\frac{\partial \mathcal{K}(q, \epsilon)}{\partial \epsilon}\right|_{\epsilon=1} .
$$

The solution of the integral in Eq. (21) is given by

$$
\begin{aligned}
\mathcal{K}(q, \epsilon)= & \frac{\Theta(\operatorname{Im} q)}{\left\langle J^{2}\right\rangle_{J} \operatorname{Im}\langle G\rangle_{\infty}} \delta\left(\operatorname{Re} q-\operatorname{Im} q \frac{\operatorname{Re}\langle G\rangle_{\infty}}{\operatorname{Im}\langle G\rangle_{\infty}}\right) \\
& \times \exp \left(-\frac{\epsilon \operatorname{Im} q}{\left\langle J^{2}\right\rangle_{J} \operatorname{Im}\langle G\rangle_{\infty}}\right),
\end{aligned}
$$

leading to the analytic expressions

$$
\begin{aligned}
\mathcal{W}(q)= & \frac{\Theta(\operatorname{Im} q)}{\left\langle J^{2}\right\rangle_{J} \operatorname{Im}\langle G\rangle_{\infty}} \delta\left(\operatorname{Re} q-\operatorname{Im} q \frac{\operatorname{Re}\langle G\rangle_{\infty}}{\operatorname{Im}\langle G\rangle_{\infty}}\right) \\
& \times \exp \left(-\frac{\operatorname{Im} q}{\left\langle J^{2}\right\rangle_{J} \operatorname{Im}\langle G\rangle_{\infty}}\right), \\
\mathcal{W}_{\text {cav }}(q)= & \frac{\Theta(\operatorname{Im} q) \operatorname{Im} q}{\left[\left\langle J^{2}\right\rangle_{J} \operatorname{Im}\langle G\rangle_{\infty}\right]^{2}} \delta\left(\operatorname{Re} q-\operatorname{Im} q \frac{\operatorname{Re}\langle G\rangle_{\infty}}{\operatorname{Im}\langle G\rangle_{\infty}}\right) \\
& \times \exp \left(-\frac{\operatorname{Im} q}{\left\langle J^{2}\right\rangle_{J} \operatorname{Im}\langle G\rangle_{\infty}}\right),
\end{aligned}
$$

where $\Theta(.$.$) is the Heaviside step function.$

As in the previous subsection, Eqs. (23) and (24) depend on $\langle G\rangle_{\infty}$, but this quantity can be determined through Eq. (9). Substituting $\mathcal{W}_{\text {cav }}(q)$ in Eq. (9) and calculating the integral, we get

$$
\begin{aligned}
\langle G\rangle_{\infty}= & \frac{z}{\left\langle J^{2}\right\rangle_{J}^{2}\langle G\rangle_{\infty}^{2}} \exp \left(-\frac{z}{\left\langle J^{2}\right\rangle_{J}\langle G\rangle_{\infty}}\right) \\
& \times \operatorname{Ei}\left(\frac{z}{\left\langle J^{2}\right\rangle_{J}\langle G\rangle_{\infty}}\right)-\frac{1}{\left\langle J^{2}\right\rangle_{J}\langle G\rangle_{\infty}},
\end{aligned}
$$

with $\operatorname{Ei}(. .$.$) denoting the complex exponential integral func-$ tion [53]. The solutions of the above equation yield the dense limit of the averaged resolvent $\langle G\rangle_{\infty}$ on the cavity graph [36,50]. The expression for $\rho(\lambda)$ in terms of $\langle G\rangle_{\infty}$ follows in an analogous way, i.e., one inserts Eq. (23) in Eq. (11) and 
calculates the remainder integral

$$
\begin{aligned}
\rho(\lambda)= & \frac{1}{\pi} \lim _{\eta \rightarrow 0^{+}} \operatorname{Im}\left[\frac{1}{\left\langle J^{2}\right\rangle_{J}\langle G\rangle_{\infty}} \exp \left(-\frac{z}{\left\langle J^{2}\right\rangle_{J}\langle G\rangle_{\infty}}\right)\right. \\
& \left.\times \operatorname{Ei}\left(\frac{z}{\left\langle J^{2}\right\rangle_{J}\langle G\rangle_{\infty}}\right)\right],
\end{aligned}
$$

with $z=\lambda-i \eta$. It is suitable to introduce the dimensionless variable $\gamma(z) \equiv \frac{z}{\left\langle J^{2}\right\rangle_{J}\langle G\rangle_{\infty}}$, in terms of which the spectral density is given by

$$
\rho(\lambda)=\frac{1}{\pi} \lim _{\eta \rightarrow 0^{+}} \operatorname{Im}\left(\frac{z^{2}+\gamma^{2}\left\langle J^{2}\right\rangle_{J}}{z \gamma^{2}\left\langle J^{2}\right\rangle_{J}}\right),
$$

where $\gamma(z)$ solves the following equation:

$$
\left\langle J^{2}\right\rangle_{J} \gamma=\frac{z^{2}}{\gamma^{2} e^{-\gamma} \operatorname{Ei}(\gamma)-\gamma} .
$$

Equations (27) and (28) constitute one of the main results of this paper. In contrast to the resolvent Eq. (19), whose analytic solution yields the Wigner semicircle law, the fixedpoint Eq. (28) for the $c \rightarrow \infty$ limit of exponential graphs has no analytic solution for arbitrary $z$. The spectral density obtained from the numerical solutions of Eq. (28) is shown in Fig. 3(a), together with direct diagonalization of large adjacency matrices of exponential random graphs with $c=100$. The agreement between these two different approaches is excellent, confirming the exactness of Eqs. (27) and (28).

Figure 3(a) suggests that $\rho(\lambda)$ diverges at $\lambda=0$. To study the singular behavior of $\rho(\lambda)$ as $|\lambda| \rightarrow 0$, one needs to understand how $\gamma(z)$ vanishes as $|z| \rightarrow 0$. With a modest amount of foresight, we make the following ansatz:

$$
\gamma(z)=\beta_{1} \frac{z}{\sqrt{\left\langle J^{2}\right\rangle_{J}}}+\beta_{2}(z) \frac{z^{2}}{\left\langle J^{2}\right\rangle_{J}}, \quad|z| \rightarrow 0,
$$

where the function $\beta_{2}(z)$ is such that $\lim _{|z| \rightarrow 0} z^{2} \beta_{2}(z)=0$, and the coefficient $\beta_{1}$ is independent of $z$. Plugging this assumption for $\gamma(z)$ in the right hand side of Eq. (28) and expanding the result in powers of $z$ up to $O\left(z^{2}\right)$, we find that the above ansatz is consistent with Eq. (28) if $\beta_{1}$ and $\beta_{2}(z)$ are

$$
\beta_{1}= \pm i, \quad \beta_{2}(z)=-\frac{1}{2}\left[E+\ln \left(-\frac{\beta_{1} z}{\sqrt{\left\langle J^{2}\right\rangle_{J}}}\right)\right],
$$

with $E$ representing the Euler-Mascheroni constant. The last step is to substitute Eq. (29) in Eq. (27) and take the limit $\eta \rightarrow 0^{+}$, which leads to the logarithmic divergence

$$
\rho(\lambda)=-\frac{1}{\pi \sqrt{\left\langle J^{2}\right\rangle_{J}}}\left[E+\ln \left(\frac{|\lambda|}{\sqrt{\left\langle J^{2}\right\rangle_{J}}}\right)\right]
$$

for $|\lambda| \rightarrow 0$. The choice of the negative sign $\beta_{1}=-i$ ensures that $\rho(\lambda)$ is non-negative. A divergence in the spectral density of random graphs at $\lambda=0$ may appear due to different reasons. For instance, the spectrum of protein-protein interaction networks has a singularity at $\lambda=0$ due to the existence of duplicated genes [54]. In the context of solid-state physics, the density of states of a tight-binding model for a quantum particle hopping on a random graph displays a divergence at $\lambda=0$ due to the presence of localized eigenstates [55].

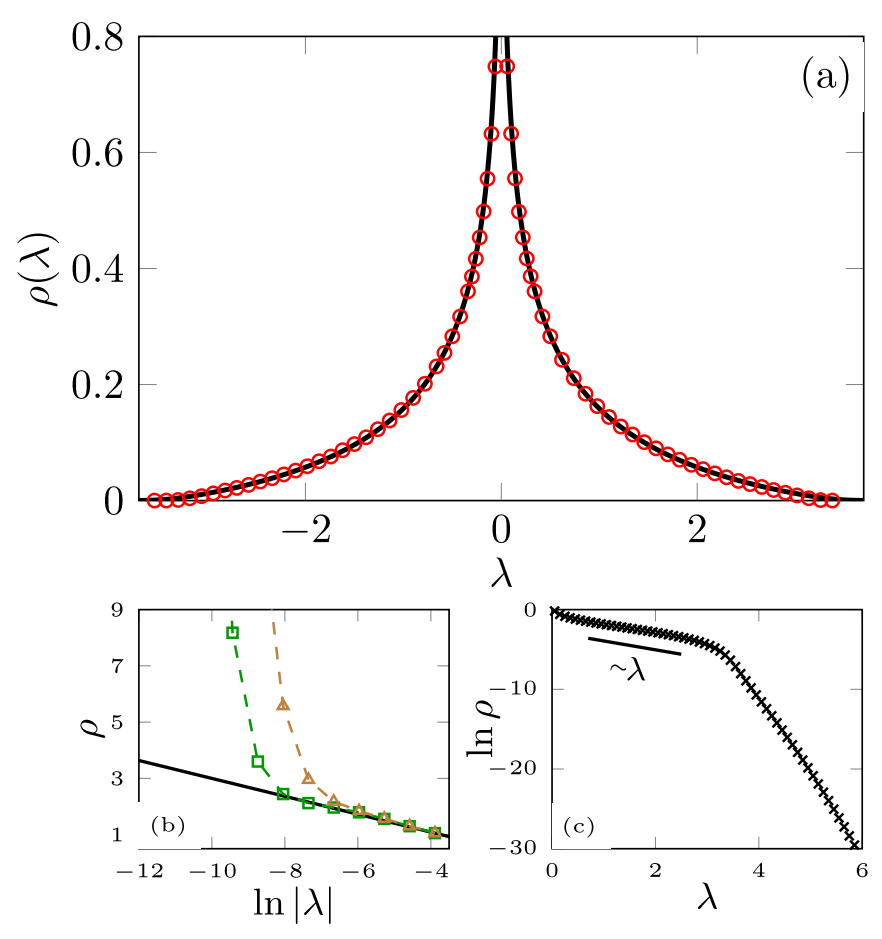

FIG. 3. Dense limit of the spectral density $\rho(\lambda)$ of random networks with an exponential degree distribution and coupling strengths drawn from $P_{J}(x)=\delta(x-1)$. (a) Comparison between the theoretical results (black solid lines), obtained from the numerical solutions of Eqs. (27) and (28) for $\eta=0$, and numerical diagonalizations (red circles) of 100 samples of $10^{4} \times 10^{4}$ adjacency matrices with $c=100$. (b) The logarithmic divergence of the spectral density for $|\lambda| \rightarrow 0$. The black solid line is the analytic result of Eq. (31), while the symbols are data derived from the numerical solutions of Eqs. (7) and (8) for $c=80$ and two values of $\eta: \eta=10^{-4}$ (brown triangles) and $\eta=10^{-5}$ (green squares). (c) The exponential behavior of the spectral density for large $|\lambda|$, obtained from the numerical solutions of Eqs. (27) and (28) for $\eta=0$.

Figure 3(b) compares Eq. (31) with the numerical solutions of the distributional Eqs. (7) and (8) for $c=80$ using a Monte Carlo method, also known as the population dynamics algorithm $[36,37,50]$. The numerical results lie on the top of the theoretical curve up to a certain $\left|\lambda_{*}\right|$, below which the numerical data for $\rho(\lambda)$ deviates from the logarithmic behavior of Eq. (31). As $\eta$ decreases, $\left|\lambda_{*}\right|$ shifts toward smaller values, confirming that the discrepancy between the numerical data and Eq. (31) is an artifact due to the finite values of $\eta$ employed in the numerical solutions of Eqs. (7) and (8).

As a final important property, we inspect the behavior of $\rho(\lambda)$ for $|\lambda| \gg 1$. As illustrated in Fig. 3(c), $\rho(\lambda)$ displays a crossover from an exponential behavior $\ln \rho(\lambda) \propto-\lambda$ to a faster decay for increasing $|\lambda|$, indicating that the spectral density does not have sharp spectral edges, but instead is supported on the entire real line. This is also consistent with a stability analysis of the fixed-point Eq. (28), which shows that the complex solution for $\gamma(z)$ remains stable for $|\lambda| \gg 1$. We point out that a perturbative study of Eq. (28) for $|\lambda| \gg 1$ is not able to capture the analytic form describing the tails of $\rho(\lambda)$. This should not come as a surprise, as the tails of $\rho(\lambda)$ 

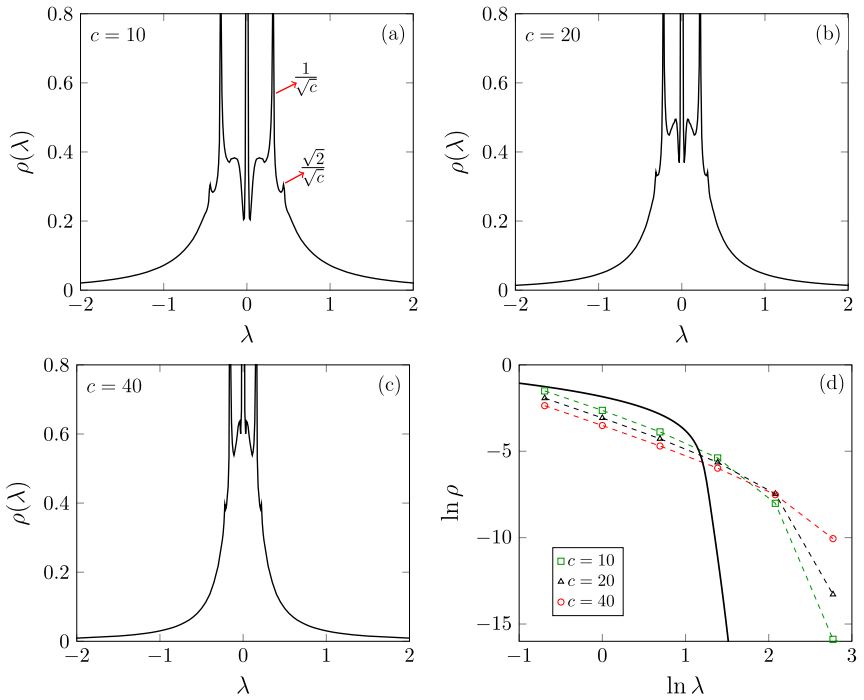

FIG. 4. The spectral density of random networks from the configuration model with a Borel degree distribution (see Table I) for different average degrees $c$ and coupling strengths drawn from $P_{J}(x)=\delta(x-1)$. All results are obtained from the numerical solutions of Eqs. (7) and (8) using the population dynamics algorithm with $\eta=10^{-3}$ (a)-(c) and $\eta=10^{-4}$ (d). Figures (a)-(c) show the spectral density of Borel random graphs for different $c$. Figure (a) also indicates the positive eigenvalues of open chains with one and two edges, and their correspondence with the position of $\delta$ peaks. Figure (d) exhibits results for the tails of $\rho(\lambda)$. The symbols are results for Borel degree distributions with different $c$, while the solid black curve shows the tail of $\rho(\lambda)$ for exponential dense random graphs as a comparison (see also Fig. 3). The dashed lines are just a guide to the eye.

are normally caused by rare statistical fluctuations in the graph structure $[34,43]$.

\section{Borel random graphs}

As a final example of network model, we present results for random networks with a Borel degree distribution (see Table I). In this case, our approach to derive analytic expressions for $\varphi(p, t)$ and $\varphi_{\text {cav }}(p, t)$ is not useful, because the probability generating function of the Borel degree distribution does not have a closed analytic form [48]. Thus, we obtain results through the numerical solution of Eqs. (7) and (8) using the population dynamics algorithm $[36,37,50]$. For Borel random graphs, the ratio $\sigma^{2} / c^{2}$ diverges as $c \rightarrow \infty$, which is precisely what renders this model interesting in comparison to Poisson and exponential graphs.

Figure 4 illustrates the evolution of the spectral density for increasing average degree. Similar to exponential random graphs, the spectral density is not described by the semicircle distribution of random matrix theory, although the values of $c$ in Fig. 4 are not large enough to observe the dense limit of $\rho(\lambda)$. We do not present results for larger values of $c$ than those in Fig. 4 because the population dynamics algorithm becomes prohibitively time consuming for increasing $c$, due to the large variance of the Borel degree distribution.

Among the distinctive features of Fig. 4, we note the existence of $\delta$ peaks in $\rho(\lambda)$. These peaks, often located at the eigenvalues of finite components [23,37,41,56], disappear for $c \rightarrow \infty$, when the fraction of nodes belonging to the giant component converges to one [1]. The spectral density in Fig. 4 displays $\delta$ peaks at the eigenvalues $\{-1 / \sqrt{c}, 1 / \sqrt{c}\}$ and $\{0,-\sqrt{2} / \sqrt{c}, \sqrt{2} / \sqrt{c}\}$ of the adjacency matrices of open chains with, respectively, two and three nodes. One can show that these $\delta$ peaks disappear in the dense limit by computing the fraction $\Pi_{s}(c)$ of nodes that belongs to a finite component with $s>1$ nodes [57]. For Poisson, exponential, and Borel degree distributions, we obtain $\Pi_{s}^{(\text {pois })}(c) \propto e^{-s c}, \Pi_{s}^{(\exp )}(c) \propto$ $c^{1-2 s}$, and $\Pi_{s}^{(\text {borel })}(c) \propto c^{1-s}$ for $c \gg 1$. Consequently, all network models studied in this paper do not have finite clusters in the dense limit. In the case of the Borel degree distribution, $\rho(\lambda)$ contains $\delta$ peaks even for very large $c$ because the function $\Pi_{s}^{(\text {borel })}(c)$ decays slower for $c \gg 1$ when compared to Poisson and exponential networks.

We have also inspected the tails of the spectral density of Borel random graphs. According to Fig. 4(d), the behavior of $\rho(\lambda)$ as a function of $\lambda$ is consistent with a power-law decay up to a certain threshold $\lambda_{p}$, above which the data clearly deviates from a straight line. As a matter of fact, we have confirmed that $\rho(\lambda)$ decreases as $\eta \rightarrow 0^{+}$for values of $\lambda$ larger than those appearing in Fig. 4(d). In spite of that, the overall tendency of the data for increasing $c$ suggests that $\rho(\lambda)$ does decay as a power law in the limit $c \rightarrow \infty$. The results in Fig. 4 are insufficient to extract the exponent characterizing the power-law tails of $\rho(\lambda)$ for $c \rightarrow \infty$, since the spectral density has not reached its limiting behavior for $c=40$. Taken together, these results indicate that $\rho(\lambda)$ is supported on the entire real line.

\section{A RIGOROUS RESULT FOR THE SPECTRAL DENSITY OF THE CONFIGURATION MODEL}

In this section, we state the main theorem of Ref. [44] and explain how the resolvent equations for $c \rightarrow \infty$, obtained in the previous section, are recovered from this theorem. We use the notation of the previous sections and we discuss the theorem in a less technical language, which helps to establish the link between Ref. [44] and the results presented in this paper in a more straightforward way.

The theorem concerns the spectral density $\rho(\lambda)$ of the adjacency matrix $\boldsymbol{A}$ [see Eq. (2)] of the configuration model in the dense limit. Let $v(\kappa)$ be the probability density of the rescaled degrees $\frac{K_{i}}{c}(i=1, \ldots, N)$ in the limit $c \rightarrow \infty$ :

$$
\nu(\kappa)=\lim _{c \rightarrow \infty} \sum_{k=0}^{\infty} p_{k} \delta\left(\kappa-\frac{k}{c}\right) .
$$

The main result of Ref. [44] is the following theorem:

Let $\left\{K_{i}\right\}_{i=1}^{N}$ be i.i.d random variables forming a degree sequence of the configuration model such that $c=o(N)$ and $J_{i j}=1$. In the limit $N \rightarrow \infty$, if the distribution of $K_{i} / c$ converges to a limit distribution $v$ with a finite second moment, then $\rho(\lambda)=\lim _{N \rightarrow \infty} \rho_{N}(\lambda)$ is given by $\nu \otimes \rho_{\mathrm{w}}$, where $\rho_{\mathrm{w}}$ is the Wigner semicircle law.

Thus, the spectral density $\rho(\lambda)$ of the configuration model with $c=o(N)$ is given by the free multiplicative convolution $\nabla$ between the probability density $v$ of rescaled degrees and the Wigner semicircle law $\rho_{\mathrm{w}}$. The free multiplicative 
convolution $v \otimes \rho_{\mathrm{w}}$ between the probability measure associated to $\rho_{\mathrm{w}}$ and the probability measure corresponding to $v$ is the unique probability measure associated to $\rho$ such that its $S$ transform $S_{\rho}(\omega)(\omega \in \mathbb{C})$ fulfills the convolution property $S_{\rho}(\omega)=S_{v}(\omega) S_{\rho_{\mathrm{w}}}(\omega)$, where $S_{v}(\omega)$ and $S_{\rho_{\mathrm{w}}}(\omega)$ are the $S$ transforms of $v$ and $\rho_{\mathrm{w}}$, respectively.

The free multiplicative convolution and the $S$ transform were introduced by Voiculescu to deal with the multiplication of free noncommuting random variables [58]. The $S$ transform finds applications in random matrix theory, since it is an important analytic tool to determine the spectral properties of products of large and independent random matrices from the spectra of the individual matrices $[59,60]$.

For unbounded measures on $\mathbb{R}_{+}$and for measures with zero mean and all moments finite, such as the Wigner semicircle distribution, the $S$ transform $S_{\varphi}(z)(z \in \mathbb{C})$ of the corresponding probability density $\varphi$ is obtained from [61,62]

$$
S_{\varphi}(z)=\frac{(1+z)}{z} \omega_{\varphi}^{-1}(z)
$$

where

$$
\omega_{\varphi}(z)=\frac{1}{z} \mathcal{C}_{\varphi}(1 / z)-1 .
$$

The function $\omega_{\varphi}^{-1}(z)$ is the functional inverse of $\omega_{\varphi}(z)$ with respect to composition and $\mathcal{C}_{\varphi}(z)$ is the Cauchy transform of $\varphi$

$$
\mathcal{C}_{\varphi}(z)=\int_{-\infty}^{\infty} \frac{d \lambda \varphi(\lambda)}{z-\lambda},
$$

where $z$ lies outside the support of $\varphi(\lambda)$. Instead of working directly with the inverse $\omega_{\varphi}^{-1}(z)$ in Eq. (33), it is more convenient to express the $S$ transform in terms of the Cauchy transform $[60,63]$. Since $\omega_{\varphi}^{-1}\left(\omega_{\varphi}(z)\right)=z$, we can rewrite Eq. (33) as follows:

$$
S_{\varphi}\left[\omega_{\varphi}(z)\right]=\frac{\left[1+\omega_{\varphi}(z)\right]}{\omega_{\varphi}(z)} z .
$$

Hence, given the $S$ transform of a probability density $\varphi$, we are able to uniquely determine $\varphi$.

In the context of random matrix theory, $\mathcal{C}_{\rho}(z)$ is the trace of the resolvent matrix

$$
\mathcal{C}_{\rho}(z)=\lim _{N \rightarrow \infty} \frac{1}{N} \sum_{i=1}^{N} G_{i i}(z),
$$

which, after setting $z=\lambda-i \eta$, gives access to the spectral density $\rho$ through Eq. (5). Combining Eqs. (5), (34), and (37), we can also express the spectral density in terms of $\omega_{\rho}(z)$ :

$$
\rho(\lambda)=\frac{1}{\pi} \lim _{\eta \rightarrow 0^{+}} \operatorname{Im}\left(\frac{\omega_{\rho}(1 / z)+1}{z}\right) .
$$

The above theorem holds for networks where $c$ scales slowly with $N$ such that $\lim _{N \rightarrow \infty} \frac{c}{N}=0$. In the previous section, we have derived results by performing the limit $c \rightarrow \infty$ after $N \rightarrow \infty$, which also implies that $\lim _{N \rightarrow \infty} \frac{c}{N}=0$. Consequently, with the exception of dense Borel networks for which $v(\kappa)$ has a divergent second moment (see Table I), the results for $\rho(\lambda)$ derived in the previous section should be recovered from the above theorem.

\section{A. Regular and Poisson random graphs}

Here we apply the theorem to degree distributions $p_{k}$ such that $\nu(\kappa)=\delta(\kappa-1)$, like regular and Poisson degree distributions. The procedure to derive the spectral density from the theorem comprises three steps: first, we compute the $S$ transforms $S_{\nu}$ and $S_{\rho_{\mathrm{w}}}$; second, we obtain the $S$ transform $S_{\rho}$ of $\rho(\lambda)$ from the convolution $S_{\rho}(\omega)=S_{v}(\omega) S_{\rho_{\mathrm{w}}}(\omega)$; third, we derive the resolvent equation from $S_{\rho}$.

Let us compute the $S$ transform of the Wigner semicircle law $\rho_{\mathrm{w}}(\lambda)$ of Eq. (20) with $\left\langle J^{2}\right\rangle_{J}=1$. The Cauchy transform $\mathcal{C}_{\rho_{\mathrm{w}}}(z)$ of $\rho_{\mathrm{w}}(\lambda)$ solves the algebraic equation [63]:

$$
\mathcal{C}_{\rho_{\mathrm{w}}}(z)=\frac{1}{z-\mathcal{C}_{\rho_{\mathrm{w}}}(z)} .
$$

Using Eq. (34) and rewriting Eq. (39) in terms of $w_{\rho_{\mathrm{w}}}(z)$, we get

$$
z= \pm \frac{\sqrt{\omega_{\rho_{\mathrm{w}}}(z)}}{1+\omega_{\rho_{\mathrm{w}}}(z)} .
$$

Inserting the above expression in Eq. (36) leads to

$$
S_{\rho_{\mathrm{w}}}\left(\omega_{\rho_{\mathrm{w}}}\right)=\frac{1}{\sqrt{\omega_{\rho_{\mathrm{w}}}}},
$$

where we have chosen the positive sign in Eq. (40). Equation (39) for the Cauchy transform of $\rho_{\mathrm{w}}$ is obtained from the $S$ transform regardless of the choice of sign in Eq. (40).

Now we turn our attention to the $S$ transform of $v(\kappa)=$ $\delta(\kappa-1)$. The Cauchy transform $\mathcal{C}_{v}(z)$ of $v(\kappa)$ reads

$$
\mathcal{C}_{v}(z)=\frac{1}{z-1}
$$

Combining Eqs. (34) and (42), we get

$$
z=\frac{\omega_{v}(z)}{1+\omega_{v}(z)},
$$

which leads to

$$
S_{v}(\omega)=1
$$

after substitution in Eq. (36). According to the above theorem, the $S$ transform of the spectral density reads

$$
S_{\rho}(\omega)=S_{\nu}(\omega) S_{\rho_{\mathrm{w}}}(\omega)=\frac{1}{\sqrt{\omega}},
$$

which immediately implies that $\rho(\lambda)$ is given by the semicircle distribution, Eq. (20). We conclude that the spectral density of dense random networks for which the distribution of the rescaled degrees $K_{i} / c$ converges to a $\delta$ peak is given by Eq. (20).

\section{B. Exponential random graphs}

For random graphs with an exponential degree distribution, $\nu(\kappa)$ reads

$$
\nu(\kappa)= \begin{cases}e^{-\kappa}, & \text { if } \kappa \geqslant 0 \\ 0, & \text { if } \kappa<0 .\end{cases}
$$

The transform $S_{\rho_{\mathrm{w}}}(\omega)$ is given by Eq. (41), hence we just need to obtain $S_{v}(\omega)$. We combine the Cauchy transform of 
Eq. (46),

$$
\mathcal{C}_{v}(z)=\int_{0}^{\infty} d \kappa \frac{e^{-\kappa}}{(z-\kappa)}=e^{-z} \operatorname{Ei}(z),
$$

with Eq. (34), obtaining

$$
\omega_{\nu}(z)=\frac{e^{-1 / z}}{z} \operatorname{Ei}(1 / z)-1
$$

To derive an explicit expression for $S_{v}\left(\omega_{v}\right)$ from Eq. (36), we have to invert analytically the above equation and find $z=$ $\phi\left(\omega_{\nu}\right)$. The analytic form of $\phi\left(\omega_{\nu}\right)$ is usually obtained when the Cauchy transform has a polynomial form [63], as in the case of the Wigner semicircle distribution. Unfortunately, here this is not the case, but we can still obtain from Eq. (48) a self-consistent equation for the inverse function $\phi\left(\omega_{\nu}\right)$,

$$
\phi\left(\omega_{v}\right)=\frac{1}{\omega_{v}} \exp \left[-\frac{1}{\phi\left(\omega_{v}\right)}\right] \operatorname{Ei}\left[\frac{1}{\phi\left(\omega_{v}\right)}\right]-\frac{\phi\left(\omega_{v}\right)}{\omega_{v}},
$$

while the $S$ transform follows from Eq. (36),

$$
S_{v}\left(\omega_{\nu}\right)=\frac{\left(1+\omega_{\nu}\right)}{\omega_{\nu}} \phi\left(\omega_{\nu}\right)
$$

with $\omega_{v}=\omega_{v}(z)$. The solution of Eq. (49) determines $S_{v}\left(\omega_{v}\right)$ through Eq. (50).

Now we are ready to recover the equations determining $\rho(\lambda)$ of exponential random graphs using the theorem of Ref. [44]. The $S$ transform of $\rho(\lambda)$ follows from the convolution

$$
S_{\rho}\left(\omega_{\rho}\right)=S_{v}\left(\omega_{\rho}\right) S_{\rho_{\mathrm{w}}}\left(\omega_{\rho}\right)=\frac{\left(1+\omega_{\rho}\right)}{\omega_{\rho} \sqrt{\omega_{\rho}}} \phi\left(\omega_{\rho}\right) .
$$

From Eq. (36), we conclude that $\phi\left(\omega_{\rho}\right)$ must also fulfill

$$
z \sqrt{\omega_{\rho}}=\phi\left(\omega_{\rho}\right)
$$

for $z$ outside the support of $\rho(\lambda)$. Substituting the above relation in Eq. (49), we find an equation that determines $\omega_{\rho}(1 / z)$ :

$$
\begin{aligned}
\frac{\sqrt{\omega_{\rho}(1 / z)}}{z}= & \frac{1}{\omega_{\rho}(1 / z)} \exp \left[-\frac{z}{\sqrt{\omega_{\rho}(1 / z)}}\right] \operatorname{Ei}\left[\frac{z}{\sqrt{\omega_{\rho}(1 / z)}}\right] \\
& -\frac{1}{z \sqrt{\omega_{\rho}(1 / z)}} .
\end{aligned}
$$

The solution for $\omega_{\rho}(1 / z)$ at $z=\lambda-i \eta$ allows us to compute the spectral density using Eq. (38). Setting

$$
\gamma(z)=\frac{z}{\sqrt{\omega_{\rho}(1 / z)}},
$$

we conclude that Eqs. (38) and (53) are identical to Eqs. (27) and (28), respectively. We also identify $\sqrt{\omega_{\rho}(1 / z)}=\langle G\rangle_{\infty}$ as the averaged resolvent on the cavity graph.

\section{THE FOURTH MOMENT OF THE EIGENVALUE DISTRIBUTION}

The results of Secs. IV and V show that the dense limit of $\rho(\lambda)$ depends on the degree distribution of the configuration model and the scope of the Wigner semicircle law is limited to graphs where $p_{k}$ becomes highly concentrated around the mean degree for $c \rightarrow \infty$. In this section, we derive a simple equation that reveals the influence of degree fluctuations on the spectral density, giving an exact condition for the breakdown of the Wigner semicircle law. We end up this section by proposing a classification of the different universal behaviors of the dense limit of $\rho(\lambda)$ for the configuration model of networks.

It is natural to characterize the statistics of random variables by computing the ratio between their moments. In the case of locally treelike random networks, the odd moments of $\rho(\lambda)$ are zero and the simplest dimensionless parameter of this type is the kurtosis $K_{N}(c)$,

$$
K_{N}(c)=\frac{\int_{-\infty}^{\infty} d \lambda \lambda^{4} \rho_{N}(\lambda)}{\left[\int_{-\infty}^{\infty} d \lambda \lambda^{2} \rho_{N}(\lambda)\right]^{2}}=\frac{N \operatorname{Tr} A^{4}}{\left(\operatorname{Tr} A^{2}\right)^{2}},
$$

where we used the definition of the empirical spectral measure, Eq. (3). When $J_{i j}=\sqrt{c} \forall i, j$, the trace $\operatorname{Tr} A^{n}(n=$ $2,3, \ldots$ ) is the total number of closed walks of length $n$ in the graph, where the length of a walk between nodes $i$ and $j$ is the number of edges that a walker traverses when going from one node to the other [18]. The relation between the moments of $\rho_{N}(\lambda)$ and $\operatorname{Tr} \boldsymbol{A}^{n}$ is very important in spectral graph theory, as it connects the eigenvalue statistics with the network structural properties [18]. The limit $N \rightarrow \infty$ must be taken before the $c \rightarrow \infty$ limit.

The trace of the second power of the adjacency matrix $\boldsymbol{A}$ reads

$$
\operatorname{Tr} \boldsymbol{A}^{2}=\frac{1}{c} \sum_{i=1}^{N} \sum_{j \in \partial_{i}} J_{i j}^{2},
$$

where $\partial_{i}$ represents the set of nodes connected to $i$. For $N \rightarrow$ $\infty$, we obtain

$$
\lim _{N \rightarrow \infty} \frac{1}{N} \operatorname{Tr} \boldsymbol{A}^{2}=\frac{1}{c}\left\langle\sum_{j \in \partial_{i}} J_{i j}^{2}\right\rangle_{J, K}=\left\langle J^{2}\right\rangle_{J},
$$

with $\langle\ldots\rangle_{J, K}$ denoting the ensemble average over the degrees and the coupling strengths. The trace of the fourth power can be written as

$$
\begin{aligned}
c^{2} \operatorname{Tr} A^{4}= & 2 \sum_{i=1}^{N} \sum_{j, r \in \partial_{i}} J_{i j}^{2} J_{r i}^{2}-\sum_{i=1}^{N} \sum_{j \in \partial_{i}} J_{i j}^{4} \\
& +\sum_{i=1}^{N} \sum_{j \in \partial_{i}} \sum_{k \in \partial_{j} \backslash i} \sum_{r \in \partial_{k} \backslash j} C_{i r} J_{i j} J_{j k} J_{k r} J_{r i},
\end{aligned}
$$

where $\partial_{j} \backslash i$ is the set of nodes adjacent to $j$, except for node $i \in \partial_{j}$. In the limit $N \rightarrow \infty$, the configuration model has a local treelike structure, cycles of length four are rare, and hence the last term on the right hand side of Eq. (58) gives only a subleading contribution, which can be neglected for $N \rightarrow \infty$, yielding the expression

$$
\lim _{N \rightarrow \infty} \frac{1}{N} \operatorname{Tr} \boldsymbol{A}^{4}=\frac{2}{c^{2}}\langle K(K-1)\rangle_{K}\left\langle J^{2}\right\rangle_{J}^{2}+\frac{1}{c}\left\langle J^{4}\right\rangle_{J} .
$$

Thus, we obtain an exact analytic expression for the $N \rightarrow \infty$ limit of the kurtosis,

$$
K_{\infty}(c)=\frac{1}{c} \frac{\left\langle J^{4}\right\rangle_{J}}{\left\langle J^{2}\right\rangle_{J}^{2}}+\frac{2}{c}(c-1)+2 \frac{\sigma^{2}}{c^{2}},
$$


valid for arbitrary degree distributions with first and second moments finite. Note that $K_{\infty}(c)$ is invariant under a rescaling of the adjacency matrix elements. Equation (60) shows how the kurtosis of the eigenvalue distribution is linked to the degree fluctuations.

Let us now establish some general conclusions about the dense limit of $\rho(\lambda)$. For $c \rightarrow \infty, K_{\infty}(c)$ behaves as

$$
\lim _{c \rightarrow \infty} K_{\infty}(c)=K_{\mathrm{w}}\left(1+\lim _{c \rightarrow \infty} \frac{\sigma^{2}}{c^{2}}\right),
$$

where $K_{\mathrm{w}}=2$ is the kurtosis of the Wigner semicircle distribution, Eq. (20). The kurtosis of the distribution $\rho_{\mathrm{w}}(\lambda)$ follows from its moments, which are given by the Catalan numbers [64]. Equation (61) unveils the central role of the degree fluctuations to the $c \rightarrow \infty$ limit of $\rho(\lambda)$. It follows that

$$
\lim _{c \rightarrow \infty} \frac{\sigma^{2}}{c^{2}}>0
$$

is a sufficient condition for the breakdown of the Wigner semicircle law. In the previous section, we have shown that $\rho(\lambda)$ converges to the semicircle distribution when the distribution $v$ of rescaled degrees is a Dirac $\delta$ peak, which is fully consistent with Eq. (62). One naturally expects that the eigenvalue statistics of network models that fulfill condition Eq. (62) is not described by the traditional results of random matrix theory.

Based on Eq. (61), we can also put forward a classification of the different universal behaviors of $\rho(\lambda)$ in the dense limit. Let us consider weighted undirected networks, defined by the adjacency matrix of Eq. (2), in which the variance of $p_{k}$ scales as $\sigma^{2}=H c^{\alpha}$ for large $c$, with $H>0$ and $\alpha$ arbitrary parameters. For this broad class of network models, the different universal behaviors of $\rho(\lambda)$ can be classified in terms of the exponent $\alpha$ that quantifies the strength of the degree fluctuations. For $\alpha<2$, the relative variance $\sigma^{2} / c^{2}$ vanishes for $c \rightarrow \infty$, which is a strong indication that $p_{k}$ is highly peaked around $c$. Thus, we obtain $\lim _{c \rightarrow \infty} K_{\infty}(c)=2$ for $\alpha<2$, and it is reasonable to conjecture that the dense limit of $\rho(\lambda)$ is given by the Wigner semicircle law. Regular and Poisson random graphs are the main examples of this class of random networks characterized by $\alpha<2$. For $\alpha>2$, the limit $\lim _{c \rightarrow \infty} K_{\infty}(c)$ diverges and we expect $\rho(\lambda)$ decays as a power-law $\rho(\lambda) \sim|\lambda|^{-\beta}$ for $|\lambda| \gg 1$, with an exponent $3<\beta<5$. The lower bound on $\beta$ is due to the finite variance of $\rho(\lambda)$ in the dense limit [see Eq. (57)]. Borel random graphs are characterized by $\alpha>2$. Finally, network models with $\alpha=2$ have a finite kurtosis $\lim _{c \rightarrow \infty} K_{\infty}(c)=2(1+H)>$ $K_{\mathrm{w}}$, whose precise value is determined by the prefactor $H$. Exponential random networks belong to this latter class where $\alpha=2$ and $H=1$. The results presented in Sec. IV are entirely consistent with this classification scheme.

\section{FINAL REMARKS}

Traditional random-matrix theory deals with the spectral properties of large random matrices with independent and identically distributed elements, providing a theoretical framework to address the universal properties of large interacting systems [65]. It is widely known that the eigenvalue distribution of undirected random networks strongly deviates from the Wigner semicircle law of random matrix theory in the sparse regime $[19,20,34,36,37,41,56]$, i.e., when the average degree $c$ is finite. As $c$ grows to infinity, random networks gradually become more fully connected and one may expect that random matrix theory describes well the spectral properties of dense random networks. We have shown in this paper that this is generally not the case.

We have studied the spectral density of the adjacency matrix of networks drawn from the configuration model with four distinct degree distributions. Our main conclusion is that the dense limit of the spectral density is governed by the strength of the degree fluctuations. It turns out that the semicircle distribution of random-matrix theory is recovered only when the degree distribution becomes, in the dense limit, highly concentrated around the mean degree. We have also derived an exact relation between the fourth moment of the eigenvalue distribution and the variance of the degree distribution, from which a sufficient condition for the breakdown of the Wigner semicircle law follows. We point out that the degree distributions considered in this paper have exponentially decaying tails, implying that all moments of the degree distribution are finite (the moments diverge only in the dense limit).

From the results derived in this paper, one expects that, in general, the circular law of random-matrix theory $[4,38]$ should also fail in describing the spectral density of directed random networks in the dense limit. Following the techniques discussed in Secs. IV and VI, the study of the eigenvalue distribution of directed networks in the dense limit is just around the corner.

Among the results in Sec. IV, we highlight the analytic equation for the averaged resolvent [see Eq. (25)], which determines the spectral density of exponential random graphs in the dense limit. This equation has no analytic solution, in contrast to the analogous Eq. (19) yielding the Wigner semicircle distribution. We have derived important features of the spectral density of dense exponential graphs, such as the existence of a logarithmic singularity around the origin and the absence of sharp spectral edges. In the case of dense random networks with a Borel degree distribution, we have studied the spectral density by solving numerically the exact Eqs. (7) and (8) for large values of the average degree. The results in Fig. 4 indicate that the spectral density of dense Borel networks is also supported on the entire real line, exhibiting power-law tails for large eigenvalues. Taken together, these results reveal remarkable differences with respect to the Wigner semicircle law.

We have shown how the analytic results of Sec. IV are recovered from the main theorem in Ref. [44]. While the results of Sec. IV follow from the equations for the distribution of the resolvent, the theorem in Ref. [44] is proved through a series of techniques, including tools from free probability theory. These two approaches are fundamentally different and we hope that our work stimulates research toward a rigorous proof of their equivalence for arbitrary degree distributions. We also remark that, although the theorem in Ref. [44] is a compelling result about the spectral density, the approach of Sec. IV is also very interesting, since it allows to compute the distribution of the resolvent, the distribution of the self-energy [66], and the inverse participation ratio [50] in the dense limit. All these quantities are important, for instance, to study the localization properties of eigenvectors. 
Based on Eq. (61) for the fourth moment of the eigenvalue distribution, we have put forward a classification scheme of the different universal behaviors of the spectral density in the dense regime. The classification holds for network models in which the variance $\sigma^{2}$ of the degree distribution scales as $\sigma^{2} \propto c^{\alpha}$ for $c \gg 1$. Networks with $\alpha<2$ exhibit weak degree fluctuations and we have conjectured that the spectral density converges to the Wigner semicircle distribution for $c \rightarrow \infty$. Networks with $\alpha>2$ display strong degree fluctuations and the spectral density is characterized by a divergent fourth moment and power-law tails for $c \rightarrow \infty$. Finally, the spectral density of dense networks with $\alpha=2$ has a finite fourth moment, whose value is larger than in the case of the Wigner semicircle distribution. The results for the specific degree distributions in Sec. IV are entirely consistent with this classification scheme. In light of this, it would be interesting to design a network model with a degree distribution that has finite moments and interpolates among the different classes.

Overall, our results shed light on the fundamental role of the degree fluctuations in the dense limit of random networks. In this context, it would be interesting to inspect the role of degree fluctuations in the local spectral properties [67-69] of dense random networks, since the local statistics of the spectrum usually exhibits a higher level of universality in comparison to the global statistics. The condition for the breakdown of the Wigner semicircle law, Eq. (62), should also apply beyond the realm of network spectra, indicating whether the degree fluctuations are strong enough to cause the failure of classic mean-field models on dense networks, such as the Curie-Weiss model [49] and the SherringtonKirkpatrick model [70]. Thus, we expect our results will stimulate research toward a better understanding of the dense limit of various models defined on random graphs, including ferromagnetic and spin-glass models [71], social dynamics [72], neural networks [73], and synchronization [8].

\section{ACKNOWLEDGMENTS}

We thank an anonymous referee for pointing out Ref. [44], which led to Sec. V. F.L.M. thanks London Mathematical Laboratory and $\mathrm{CNPq} /$ Brazil for financial support. J.D.S. acknowledges a fellowship from the London Mathematical Laboratory.
[1] M. Newman, Networks: An Introduction (Oxford University Press, Oxford, UK, 2010).

[2] S. Bornholdt and H. G. Schuster, Handbook of Graphs and Networks: From the Genome to the Internet (Wiley, Weinheim, Germany, 2003).

[3] A. Barrat, M. Barthélemy, and A. Vespignani, Dynamical Processes on Complex Networks (Cambridge University Press, Cambridge, UK, 2008).

[4] F. L. Metz, I. Neri, and T. Rogers, Spectral theory of sparse non-hermitian random matrices, J. Phys. A: Math. Theor. 52, 434003 (2019).

[5] I. Neri and F. L. Metz, Linear stability analysis of large dynamical systems on random directed graphs, Phys. Rev. Res. 2, 033313 (2020).

[6] W. Tarnowski, I. Neri, and P. Vivo, Universal transient behavior in large dynamical systems on networks, Phys. Rev. Res. 2, 023333 (2020).

[7] R. Pastor-Satorras, C. Castellano, P. Van Mieghem, and A. Vespignani, Epidemic processes in complex networks, Rev. Mod. Phys. 87, 925 (2015).

[8] J. G. Restrepo, E. Ott, and B. R. Hunt, Onset of synchronization in large networks of coupled oscillators, Phys. Rev. E 71, 036151 (2005).

[9] A. Arenas, A. Díaz-Guilera, J. Kurths, Y. Moreno, and C. Zhou, Synchronization in complex networks, Phys. Rep. 469, 93 (2008).

[10] Y. Chen, Y. Shen, P. Lin, D. Tong, Y. Zhao, S. Allesina, $\mathrm{X}$. Shen, and C.-I. Wu, Gene regulatory network stabilized by pervasive weak repressions: MicroRNA functions revealed by the May-Wigner theory, Natl. Sci. Rev. 6, 1176 (2019).

[11] Y. Guo and A. Amir, Stability of gene regulatory networks, arXiv:2006.00018 [physics.bio-ph].

[12] R. M. May, Will a large complex system be stable? Nature 238, 413 (1972).
[13] S. Allesina, J. Grilli, György Barabás, S. Tang, and J. Aljadeff, Predicting the stability of large structured food webs, Nat. Commun. 6, 7842 (2015).

[14] J. Grilli, T. Rogers, and S. Allesina, Modularity and stability in ecological communities, Nat. Commun. 7, 12031 (2016).

[15] H. Sompolinsky, A. Crisanti, and H. J. Sommers, Chaos in Random Neural Networks, Phys. Rev. Lett. 61, 259 (1988).

[16] K. Rajan and L. F. Abbott, Eigenvalue Spectra of Random Matrices for Neural Networks, Phys. Rev. Lett. 97, 188104 (2006).

[17] F. Schuessler, A. Dubreuil, F. Mastrogiuseppe, S. Ostojic, and O. Barak, Dynamics of random recurrent networks with correlated low-rank structure, Phys. Rev. Res. 2, 013111 (2020).

[18] P. van Mieghem, Graph Spectra for Complex Networks (Cambridge University Press, Cambridge, UK, 2012).

[19] Illés J. Farkas, I. Derényi, Albert-László Barabási, and Tamás Vicsek, Spectra of "real-world" graphs: Beyond the semicircle law, Phys. Rev. E 64, 026704 (2001).

[20] S. N. Dorogovtsev, A. V. Goltsev, J. F. F. Mendes, and A. N. Samukhin, Spectra of complex networks, Phys. Rev. E 68, 046109 (2003).

[21] G. J. Rodgers, K Austin, B Kahng, and D Kim, Eigenvalue spectra of complex networks, J. Phys. A: Math. Gen. 38, 9431 (2005).

[22] T. Rogers, C. P. Vicente, K. Takeda, and I. P. Castillo, Spectral density of random graphs with topological constraints, J. Phys. A: Math. Theor. 43, 195002 (2010).

[23] R. Kühn and J. van Mourik, Spectra of modular and small-world matrices, J. Phys. A: Math. Theor. 44, 165205 (2011).

[24] F. L. Metz, I. Neri, and D. Bollé, Spectra of sparse regular graphs with loops, Phys. Rev. E 84, 055101(R) (2011).

[25] M. E. J. Newman, Spectra of networks containing short loops, Phys. Rev. E 100, 012314 (2019).

[26] M. Molloy and B. Reed, A critical point for random graphs with a given degree sequence, Random Struct. Algorithms 6, 161 (1995). 
[27] M. Molloy and B. Reed, The size of the giant component of a random graph with a given degree sequence, Comb. Probab. Comput. 7, 295 (1998).

[28] M. E. J. Newman, S. H. Strogatz, and D. J. Watts, Random graphs with arbitrary degree distributions and their applications, Phys. Rev. E 64, 026118 (2001).

[29] R. Solomonoff and A. Rapoport, Connectivity of random nets, Bull. Math. Biophys. 13, 107 (1951).

[30] P. Erdös and A. Rényi, On random graphs I., Publ. Math. Debrecen 6, 290 (1959).

[31] P. Erdös and A. Rényi, On the evolution of random graphs, Publ. Math. Inst. Hungarian Acad. Sci. 5, 17 (1960).

[32] Réka Albert and Albert-László Barabási, Statistical mechanics of complex networks, Rev. Mod. Phys. 74, 47 (2002).

[33] A. Clauset, C. R. Shalizi, and M. E. J. Newman, Power-law distributions in empirical data, SIAM Rev. 51, 661 (2009).

[34] G. J. Rodgers and A. J. Bray, Density of states of a sparse random matrix, Phys. Rev. B 37, 3557 (1988).

[35] K.-I. Goh, B. Kahng, and D. Kim, Spectra and eigenvectors of scale-free networks, Phys. Rev. E 64, 051903 (2001).

[36] T. Rogers, I. P. Castillo, R. Kühn, and K. Takeda, Cavity approach to the spectral density of sparse symmetric random matrices, Phys. Rev. E 78, 031116 (2008).

[37] R. Kühn, Spectra of sparse random matrices, J. Phys. A: Math. Theor. 41, 295002 (2008).

[38] T. Rogers and I. P. Castillo, Cavity approach to the spectral density of non-hermitian sparse matrices, Phys. Rev. E 79, 012101 (2009).

[39] I. Neri and F. L. Metz, Spectra of Sparse Non-Hermitian Random Matrices: An Analytical Solution, Phys. Rev. Lett. 109, 030602 (2012).

[40] F. L. Metz and I. Pérez Castillo, Large Deviation Function for the Number of Eigenvalues of Sparse Random Graphs Inside an Interval, Phys. Rev. Lett. 117, 104101 (2016).

[41] M. E. J. Newman, X. Zhang, and R. R. Nadakuditi, Spectra of random networks with arbitrary degrees, Phys. Rev. E 99, 042309 (2019).

[42] C. Bordenave and M. Lelarge, Resolvent of large random graphs, Random Struct. Algorithms 37, 332 (2010).

[43] G. Semerjian and L. F. Cugliandolo, Sparse random matrices: The eigenvalue spectrum revisited, J. Phys. A: Math. Gen. 35, 4837 (2002).

[44] A. Dembo, E. Lubetzky, and Y. Zhang, Empirical spectral distributions of sparse random graphs, in In and Out of Equilibrium 3: Celebrating Vladas Sidoravicius, Progress in Probability, Vol. 77, edited by M. E. Vares, R. Fernandez, L. R. Fontes, and C. M. Newman (Birkhäuser Basel, Basel, Switzerland, 2020).

[45] G. Livan, M. Novaes, and P. Vivo, Introduction to Random Matrices: Theory and Practice, SpringerBriefs in Mathematical Physics (Springer International Publishing, Cham, Switzerland, 2018).

[46] E. Borel, C. R. Acad. Sci. 214, 452 (1942).

[47] P. C. Consul, S. Kotz, and F. Famoye, Lagrangian Probability Distributions (Birkhäuser, Boston, 2005).

[48] H. Finner, P. Kern, and M. Scheer, On some compound distributions with Borel summands, Insur.: Math. Econ. 62, 234 (2015).

[49] R. J. Baxter, Exactly Solved Models in Statistical Mechanics, Dover Books on Physics (Dover Publications, New York, 2007).

[50] F. L. Metz, I. Neri, and D. Bollé, Localization transition in symmetric random matrices, Phys. Rev. E 82, 031135 (2010).
[51] Réka Albert, István Albert, and G. L. Nakarado, Structural vulnerability of the North American power grid, Phys. Rev. E 69, 025103(R) (2004).

[52] L. A. N. Amaral, A. Scala, M. Barthélémy, and H. E. Stanley, Classes of small-world networks, Proc. Natl. Acad. Sci. 97, 11149 (2000).

[53] I. S. Gradshteyn and I. M. Ryzhik, Table of Integrals, Series, and Products (Elsevier Science, San Diego, 2014).

[54] C. Kamp and K. Christensen, Spectral analysis of proteinprotein interactions in drosophila melanogaster, Phys. Rev. E 71, 041911 (2005)

[55] S. N. Evangelou, A numerical study of sparse random matrices, J. Stat. Phys. 69, 361 (1992).

[56] M. Bauer and O. Golinelli, Random incidence matrices: Moments of the spectral density, J. Stat. Phys. 103, 301 (2001)

[57] See chapter 13 of [1].

[58] D. Voiculescu, Multiplication of certain non-commuting random variables, J. Operator Theo. 18, 223 (1987).

[59] Z. Burda, R. A. Janik, and M. A. Nowak, Multiplication law and $s$ transform for non-Hermitian random matrices, Phys. Rev. E 84, 061125 (2011).

[60] W. Młotkowski, M. A. Nowak, K. A. Penson, and K. Życzkowski, Spectral density of generalized Wishart matrices and free multiplicative convolution, Phys. Rev. E 92, 012121 (2015).

[61] N. R. Rao and R. Speicher, Multiplication of free random variables and the S-transform: The case of vanishing mean, Electron. Commun. Probab. 12, 248 (2007).

[62] H. Bercovici and D. Voiculescu, Free convolution of measures with unbounded support, Indiana Univ. Math. J. 42, 733 (1993).

[63] N. R. Rao and A. Edelman, The polynomial method for random matrices, Found. Comput. Math. 8, 649 (2008).

[64] E. P. Wigner, Characteristic vectors of bordered matrices with infinite dimensions, Ann. Math. 62, 548 (1955).

[65] G. Akemann, J. Baik, and P. Di Francesco, The Oxford Handbook of Random Matrix Theory, Oxford Handbooks in Mathematics Series (Oxford University Press, Oxford, UK, 2015).

[66] R Abou-Chacra, D. J. Thouless, and P. W. Anderson, J. Phys. C: Solid State Phys. 6, 1734 (1973).

[67] J. O. Lee and K. Schnelli, Local law and Tracy Widom limit for sparse random matrices, Probab. Theory Relat. Fields 171, 543 (2018).

[68] J. Huang, B. Landon, and H.-T. Yau, Transition from Tracy Widom to gaussian fluctuations of extremal eigenvalues of sparse Erdös-Rényi graphs, Ann. Probab. 48, 916 (2020).

[69] R. Bauerschmidt, J. Huang, A. Knowles, and H. Yau, Edge rigidity and universality of random regular graphs of intermediate degree, Geom. Funct. Anal. 30, 693 (2020).

[70] D. Sherrington and S. Kirkpatrick, Solvable Model of a SpinGlass, Phys. Rev. Lett. 35, 1792 (1975).

[71] M. Leone, A. Vázquez, A. Vespignani, and R. Zecchina, Ferromagnetic ordering in graphs with arbitrary degree distribution, Eur. Phys. J. B 28, 191 (2002).

[72] C. Castellano, S. Fortunato, and V. Loreto, Statistical physics of social dynamics, Rev. Mod. Phys. 81, 591 (2009).

[73] A. C. C. Coolen, R. Kühn, and P. Sollich, Theory of Neural Information Processing Systems (Oxford University Press, Oxford, UK, 2005). 